\title{
A más de 11 años de la implementación de la conciliación extrajudicial: ¿cómo vamos?
}

Rocio Peñafiel Garreta*

http://dx.doi.org/10.21503/lex.v10i9.338

Abogada por la Universidad Nacional Mayor de San Marcos. Egresada de la Maestría en Derecho con Mención en Política Jurisdiccional de la Pontificia Universidad Católica del Perú. Directora del Centro de Conciliación Construyendo Alternativas para la Paz (CREAPAZ). 


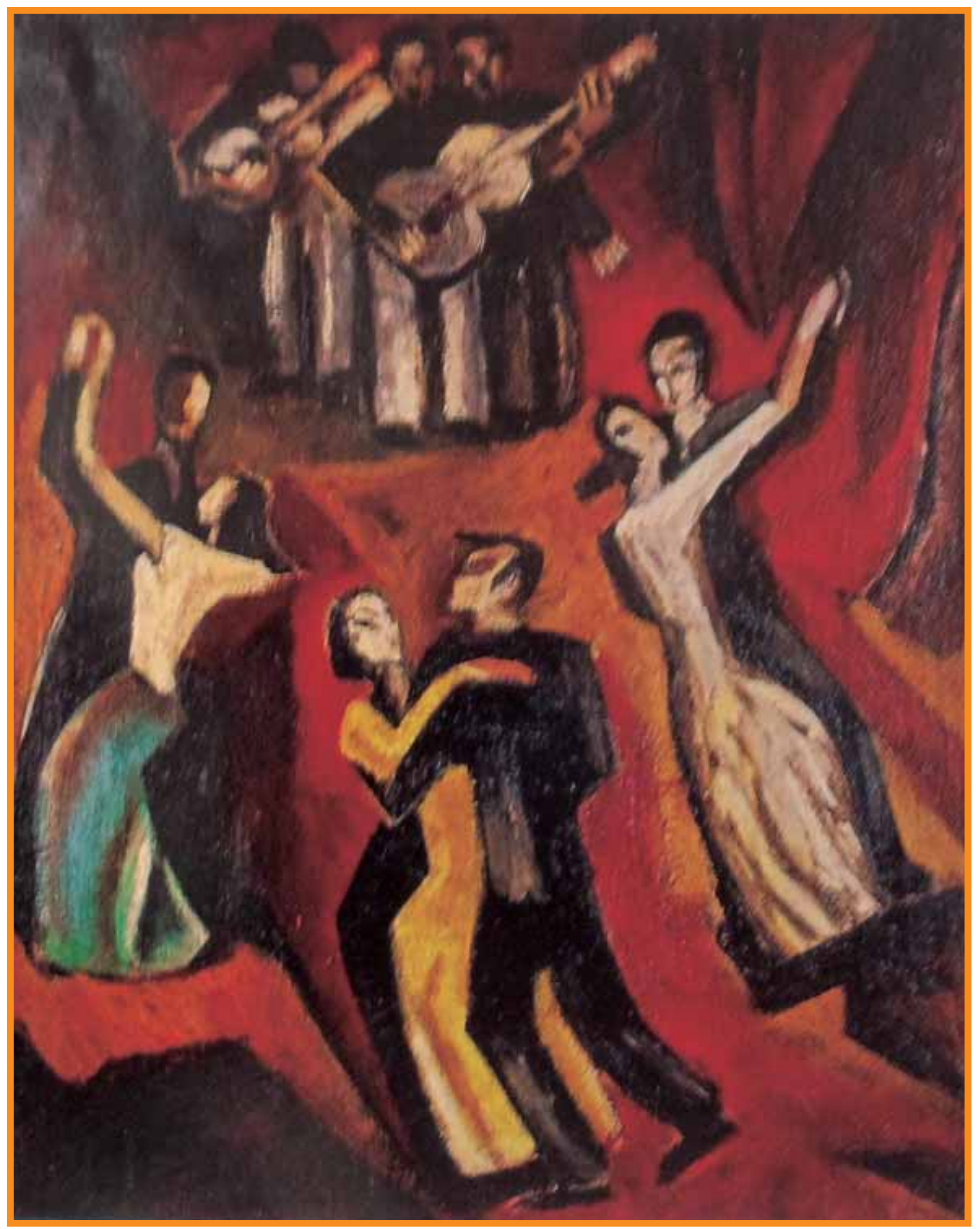

El vals. Colección particular. 
$\mathrm{E}$ n el 2008, mientras terminábamos de escribir un artículo analizando algunas cifras de la conciliación extrajudicial, ${ }^{1}$ y cuando identificábamos que uno de los problemas de esta institución era la elevada cantidad de inasistencias a las audiencias de conciliación, ${ }^{2}$ se produjeron cambios normativos orientados a propiciar una mayor asistencia de las partes a los procesos de conciliación.

El Decreto Legislativo No 1070, publicado en el diario oficial El Peruano el 28 de junio de 2008, modificó diversos artículos de la Ley de Conciliación. A decir del especialista Martín Pinedo Aubián, ${ }^{3}$ "la modificatoria más importante se ha dado en el sentido de fortalecer a la conciliación extrajudicial, al considerarla como requisito de procedibilidad en virtud de una lectura del artículo $6^{\circ}$ de la Ley...".

Como señala el mismo autor,5 "tanto el artículo $6^{\circ}$ de la Ley como la parte final del artículo $15^{\circ}$ de la Ley señalan un Régimen de Obligatoriedad de Concurrencia de las Partes a la Audiencia de Conciliación, puesto que de su tenor se desprenden varias sanciones a la parte que no concurre a la audiencia de conciliación, a saber:

1 Peñafiel Garreta, Rocío, “Conciliación extrajudicial: algunos datos para la discusión”, En: http://www.justiciayderecho. org/revista $4 /$ articulos/Conciliacion\%20 extrajudicial\%20algunos\%20datos\%20para\%20el\%20debate\%20sobre\%20 su\%20funcionamiento\%20Rocio\%20Penafiel.pdf

2 Las cifras por inasistencias ya sea de una o ambas partes a las audiencias de conciliación en materias civiles en el año 2005 , por ejemplo, llegaron al $71 \%$. Recordemos que antes de las modificaciones legislativas del Decreto Legislativo 1071, bastaba con presentar un acta en la que constara que el demandante había intentado conciliar presentando su solicitud de conciliación, no sancionándose si alguna de las partes, incluso si el mismo demandante, no asistía a la audiencia de conciliación. Esta situación cambió con el Decreto Legislativo $N^{\circ} 1071$, publicado el 28 de junio de 2008, que modificó la Ley de Conciliación.

3 Pinedo Aubián, Martín, "Evite la improcedencia de su demanda”, en: http://www.pinedomartin.blogspot.com/2012/06/ evite-la-improcedencia-de-su-demanda.html. 25 de junio de 2012.

4 Ley No 26872. Ley de Conciliación: "Artículo 6. Falta de intento conciliatorio. Si la parte demandante, en forma previa a interponer su demanda judicial, no solicita ni concurre a la audiencia respectiva ante un Centro de Conciliación extrajudicial para los fines señalados en el artículo precedente, el juez competente al momento de calificar la demanda, la declarará improcedente por causa de manifiesta falta de interés para obrar."

5 Pinedo Aubián, Martín, "Ni a favor ni en contra... ¡Todo lo contrario! ((Problemas derivados de la interpretación y aplicación del Decreto Legislativo No 1070 y su incidencia en el ámbito procesal y extraprocesal)”, febrero de 2010. En: http://www.pinedomartin.blogspot.com/2010/02/ni-favor-ni-en-contra-todo-lo-contrario.html 
I. Si la parte demandante no solicita ni concurre a la audiencia de conciliación extrajudicial, el Juez, al momento de calificar la demanda, la declarará improcedente por causa de manifiesta falta de interés para obrar.

II. Si el invitado no concurre a la audiencia, entonces no podrá formular reconvención. La misma sanción se aplica a la parte invitada que concurra a la audiencia de conciliación pero no plantee en el acta de conciliación los fundamentos de su probable reconvención.

III. La inasistencia de la parte invitada a la audiencia de conciliación produce, en el proceso judicial que se instaure, presunción legal relativa de verdad sobre los hechos expuestos en el acta de conciliación y reproducidos en la demanda.

IV. La misma presunción legal relativa de verdad se aplicará a favor del invitado que asista y exponga los hechos que determinen sus pretensiones para una probable reconvención, en el supuesto de que el solicitante no asista.

V. El Juez debe imponer en el proceso una multa no menor de dos ni mayor de diez Unidades de Referencia Procesal a la parte que no haya asistido a la Audiencia de Conciliación Extrajudicial."

Este nuevo régimen nos hizo pensar que se produciría una fuerte tendencia ascendente de las asistencias a las audiencias de conciliación y, por el contrario, habría un fuerte descenso de las inasistencias. Sin embargo, las cifras no confirman esta hipótesis, como veremos posteriormente.

Han transcurrido más de 11 años desde que la conciliación extrajudicial inició su implementación en distintos lugares, y a casi cuatro años de estos cambios legislativos orientados a propiciar una mayor concurrencia de las partes a las audiencias, la discusión de si la institución ha fracasado o por el contrario ha tenido éxito sigue vigente, ${ }^{6}$ a pesar de que sigue sin haber información suficiente que pueda hacernos arribar a una conclusión objetiva al respecto.

Para contribuir con esta discusión, tratando de hacerlo en base a información objetiva, es decir, en base a datos que nos puedan ayudar a esclarecer al menos algunos temas, decidimos actualizar nuestras cifras a través de una nueva solicitud al Ministerio de Justicia de datos

6 Por ejemplo, la doctora Eugenia Ariano Deho, en una entrevista realizada por Renzo Cavani Brain para la Revista Jurídica del Perú, en un especial sobre "El Régimen de Conciliación Extrajudicial a tres años de su reforma", afirma que no cree que este sistema funcione. Por su parte, el doctor Martín Pinedo Aubián, si bien señala que no se puede hablar de manera absoluta de un triunfo o fracaso de la institución conciliadora, sí considera que se ha logrado fortalecer el sistema conciliatorio a través de la existencia de un régimen de obligatoriedad de concurrencia de las partes que garantiza la posibilidad de realizar la audiencia de conciliación. En: Revista Jurídica del Perú, tomo 124, junio de 20011, pp. 13 a 19. 
relacionados con la cantidad de casos ingresados y resueltos en centros de conciliación tanto públicos como privados.

También se solicitó información con respecto al cumplimiento de los acuerdos plasmados en las Actas de Conciliación y sobre la cantidad de actas que son presentadas al Poder Judicial para su ejecución, al no ser cumplidos los acuerdos que ahí se plasmaron. Así mismo, se solicitaron informes de evaluación de la institución conciliatoria que puedan ayudar a analizar su desempeño. Se nos informó que el Ministerio de Justicia no contaba con información sobre estos dos últimos puntos, por lo que solo podremos analizar el funcionamiento de la institución con base en la información estadística sobre cantidad de casos ingresados y resueltos, asistencias, inasistencias a las audiencias, cantidad de actas con acuerdo, falta de acuerdo, etc.; pero, lamentablemente, una vez más, no podremos analizar si estos acuerdos son cumplidos posteriormente o no.

Veamos a continuación con detalle la información relacionada con centros privados entre los años 2001 y 2011, a fin de poder comparar esta información con la presentada en el artículo anterior en el 2008, ya que en aquella época se nos proporcionó información solo con respecto a centros privados. Más adelante analizaremos también las diferencias con los casos atendidos por los centros de conciliación públicos. Veamos:

\section{ANÁLISIS DE LA INFORMACIÓN ESTADÍSTICA A NIVEL GLOBAL (TODAS LAS MATERIAS)}

\begin{tabular}{|c|c|c|c|c|c|c|c|c|c|}
\hline \multicolumn{10}{|c|}{$\begin{array}{l}\text { CANTIDAD DE INGRESOS EN CENTROS DE CONCILIACIÓN PRIVADOS Y PORCENTAJES CON } \\
\text { RELACIÓN A TOTAL DE INGRESOS }\end{array}$} \\
\hline AÑOS & Civil & $\%$ & Familia & $\%$ & Penal & $\%$ & Otros & $\%$ & TOTAL \\
\hline 2001 & 17286 & $97,0 \%$ & 422 & $2,4 \%$ & 34 & $0,2 \%$ & 72 & $0,4 \%$ & 17814 \\
\hline 2002 & 31913 & $97,7 \%$ & 530 & $1,6 \%$ & 19 & $0,1 \%$ & 216 & $0,7 \%$ & 32678 \\
\hline 2003 & 35944 & $97,5 \%$ & 724 & $2,0 \%$ & 31 & $0,1 \%$ & 170 & $0,5 \%$ & 36869 \\
\hline 2004 & 34788 & $95,0 \%$ & 1000 & $2,7 \%$ & 39 & $0,1 \%$ & 806 & $2,2 \%$ & 36633 \\
\hline 2005 & 37717 & $95,5 \%$ & 1554 & $3,9 \%$ & 176 & $0,4 \%$ & 38 & $0,1 \%$ & 39485 \\
\hline 2006 & 35580 & $92,3 \%$ & 2583 & $6,7 \%$ & 15 & $0,0 \%$ & 388 & $1,0 \%$ & 38566 \\
\hline 2007 & 39705 & $93,1 \%$ & 2737 & $6,4 \%$ & 22 & $0,1 \%$ & 178 & $0,4 \%$ & 42642 \\
\hline 2008 & 37564 & $86,4 \%$ & 5556 & $12,8 \%$ & 64 & $0,1 \%$ & 288 & $0,7 \%$ & 43472 \\
\hline 2009 & 41895 & $77,4 \%$ & 11654 & $21,5 \%$ & 18 & $0,0 \%$ & 538 & $1,0 \%$ & 54105 \\
\hline 2010 & 41843 & $78,0 \%$ & 11180 & $20,8 \%$ & 11 & $0,0 \%$ & 600 & $1,1 \%$ & 53634 \\
\hline 2011 & 43809 & $75,6 \%$ & 13824 & $23,9 \%$ & 40 & $0,1 \%$ & 272 & $0,5 \%$ & 57945 \\
\hline TOTAL & 398044 & $87,7 \%$ & 51,764 & $11,4 \%$ & 469 & $0,1 \%$ & 3,566 & $0,8 \%$ & 453843 \\
\hline
\end{tabular}

Fuente: Elaboración propia a partir de información proporcionada por el MINJUS. 
Lo primero que observamos es que la tendencia ascendente en el número de casos atendidos se mantiene, habiéndose más que triplicado el número de casos ingresados en el 2011 con relación al año 2001. Este incremento es mucho más notorio en las materias de familia, donde se ha multiplicado en más de 30 veces, llegando a representar casi uno de cada cuatro recibidos por los centros de conciliación privados. Como veremos más adelante, esta mayor participación de los casos de familia es aún mucho más marcada en los centros públicos.

A pesar de ello, siguen siendo los casos civiles los más numerosos. Del $100 \%$ de casos analizados en el período 2008-2011, los asuntos relacionados con materias civiles siguieron representando el mayor porcentaje, al igual que sucedió entre el 2001 y 2008, aunque resulta llamativo que su participación haya ido reduciéndose de $96 \%$ (que fue el promedio de asuntos civiles recibidos en centros privados entre 2001 y 2007) a $79 \%$ (que es el promedio entre el 2008 y 2011). Como un promedio general entre los años 2001 Y 2011, tenemos que el $88 \%$ de las solicitudes ingresadas en centros privados corresponden a materias civiles, 11 $\%$ a familia y $1 \%$ a materias penales y otras.

Como se puede apreciar en el mismo cuadro, si bien los porcentajes disminuyen, esto no significa que la cantidad de casos en términos absolutos haya disminuido, sino, como se puede observar en el mismo cuadro, la proporción de materias civiles disminuye por un aumento mucho más acelerado de las materias de familia.

Si observamos en forma separada a los centros privados y a los centros públicos, resulta claro que en los centros públicos una gran mayoría de los casos ingresados son por materias relacionadas a temas de familia. Así tenemos que del total de casos de centros públicos entre los años 2008 al 2011, los casos civiles representan únicamente el 15,5\%, mientras que los casos de familia representan el $84,2 \%$ de las causas ingresadas. Entre penal, laboral y otros (conflictos vecinales, etc.), representan apenas el 0,2\%.

\begin{tabular}{|c|c|c|c|c|c|c|c|c|c|}
\hline \multicolumn{10}{|c|}{ TOTAL DE INGRESOS EN CENTROS DE CONCILIACIÓN PÚBLICOS POR MATERIA } \\
\hline AÑOS & Civil & $\%$ & Familia & $\%$ & Penal & \begin{tabular}{l|l}
$\%$ & \\
\end{tabular} & Otros & $\%$ & TOTAL \\
\hline 2008 & 2513 & $20,2 \%$ & 9894 & $79,4 \%$ & 12 & $0,1 \%$ & 47 & $0,4 \%$ & 12466 \\
\hline 2009 & 2958 & $16,8 \%$ & 14605 & $82,9 \%$ & 12 & $0,1 \%$ & 51 & $0,3 \%$ & 17626 \\
\hline 2010 & 2535 & $14,4 \%$ & 15056 & $85,4 \%$ & 2 & $0,0 \%$ & 45 & $0,3 \%$ & 17638 \\
\hline 2011 & 2108 & $12,0 \%$ & 15465 & $88,0 \%$ & 0 & $0,0 \%$ & 7 & $0,0 \%$ & 17580 \\
\hline TOTAL & 10114 & $15,5 \%$ & 55020 & $84,2 \%$ & 26 & $0,0 \%$ & 150 & $0,2 \%$ & 65,310 \\
\hline
\end{tabular}

Fuente: Elaboración propia a partir de información proporcionada por el MINJUS. 
Del mismo cuadro, tanto a partir de las cifras absolutas como porcentuales, podemos apreciar que los centros púbicos se estarían concentrando en materia familiar.

Si analizamos en forma conjunta a los centros privados y públicos entre el 2008 y 2011, encontramos que en promedio el $64 \%$ de casos corresponden a materias civiles, mientras que el 35,6\% a familia. Los casos de materia penal alcanzan únicamente el 0,1\% del total de casos atendidos, mientras que el resto (conflictos vecinales, etc.) hace un total de $0,4 \%$, como se observa en el siguiente cuadro:

\begin{tabular}{|c|c|c|c|c|c|c|c|c|c|}
\hline AÑOS & Civil & $\%$ & Familia & $\%$ & Penal & $\%$ & Otros & $\%$ & TOTAL \\
\hline 2008 & 40077 & $71,8 \%$ & 15450 & $27,7 \%$ & 76 & $0,1 \%$ & 191 & $0,3 \%$ & 55794 \\
\hline 2009 & 44853 & $62,8 \%$ & 26259 & $36,7 \%$ & 30 & $0,0 \%$ & 320 & $0,4 \%$ & 71462 \\
\hline 2010 & 44378 & $62,5 \%$ & 26236 & $37,0 \%$ & 13 & $0,0 \%$ & 345 & $0,5 \%$ & 70972 \\
\hline 2011 & 45917 & $60,9 \%$ & 29289 & $38,9 \%$ & 40 & $0,1 \%$ & 143 & $0,2 \%$ & 75389 \\
\hline TOTAL & 175225 & $64,0 \%$ & 97234 & $35,5 \%$ & 159 & $0,1 \%$ & 999 & $0,4 \%$ & 273617 \\
\hline
\end{tabular}

Fuente: Elaboración propia a partir de información proporcionada por el MINJUS.

Otro análisis relevante es el relacionado con los porcentajes de asistencias e inasistencias en los últimos años. Con base en el nuevo marco normativo antes mencionado, esperábamos una tendencia ascendente del porcentaje de asistencias; sin embargo, los datos no corroboraron esta expectativa:

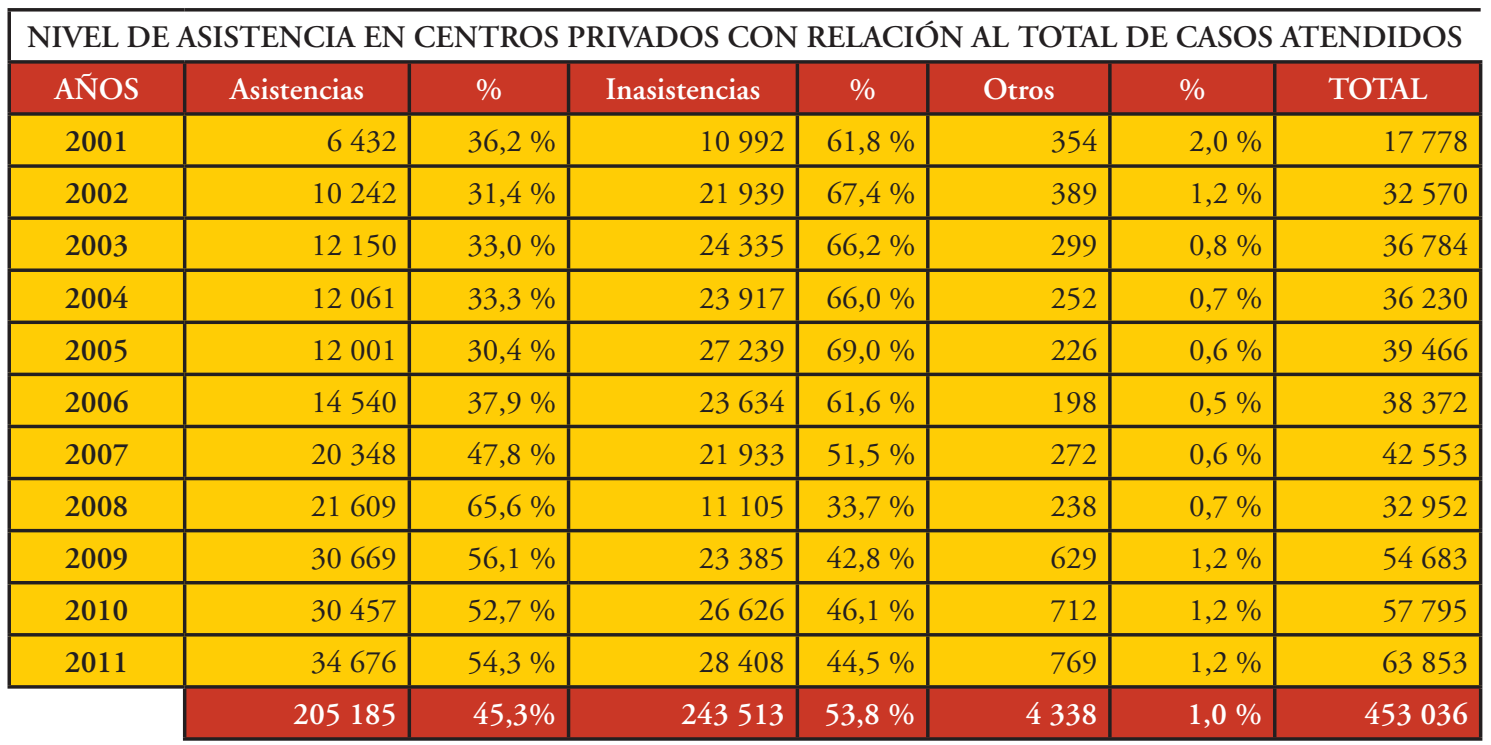

Fuente: Elaboración propia a partir de información proporcionada por el MINJUS. 
En primer lugar, se observa una disminución del porcentaje de inasistencias a partir del 2005 (año en el que alcanzó un pico de 69 \%) hasta el 2008 (año en que alcanzó su punto más bajo con solo $33 \%$, es decir, cayó a menos de la mitad en apenas 3 años). Esto podría interpretarse como una creciente confianza y apuesta por parte de los usuarios para darle una oportunidad al sistema de conciliación.

Lo que llama la atención es que, contrariamente a lo esperado, a partir del 2008, el porcentaje de inasistencias, que había decaído fuertemente a un 33,7 \%, no mantiene esta tendencia descendente en los años siguientes, sino que, por el contrario, este nuevamente aumenta a niveles de alrededor de $45 \%$ en los 3 años siguientes. No obstante ello, estos porcentajes nunca han vuelto a ser tan altos como en los primeros años.

En comparación con esos resultados, en los centros públicos, el promedio de inasistencias es menor $(33,3 \%)$, observándose una ligera tendencia a la disminución relativa (porcentual) de las asistencias.

\begin{tabular}{|c|r|r|r|r|r|r|r|}
\hline \multicolumn{6}{|c|}{ NIVEL DE ASISTENCIA EN CENTROS PÚBLICOS CON RELACIÓN AL TOTAL DE CASOS ATENDIDOS } \\
\hline ANOS & Asistencias & $\%$ & Inasistencias & \multicolumn{1}{l}{$\%$} & \multicolumn{1}{c|}{ Otros } & $\%$ & \multicolumn{1}{c|}{ TOTAL } \\
\hline 2008 & 6561 & $70,6 \%$ & 2682 & $28,9 \%$ & 46 & $0,5 \%$ & 9289 \\
\hline 2009 & 9432 & $63,1 \%$ & 5260 & $35,2 \%$ & 265 & $1,8 \%$ & 14957 \\
\hline 2010 & 9390 & $62,5 \%$ & 5378 & $35,8 \%$ & 260 & $1,7 \%$ & 15028 \\
\hline 2011 & 10,059 & $58,1 \%$ & 5518 & $31,9 \%$ & 1729 & $10,0 \%$ & 17306 \\
\hline & 35442 & $\mathbf{6 2 , 6} \%$ & 18838 & $33,3 \%$ & 2300 & $4,1 \%$ & 56580 \\
\hline
\end{tabular}

Fuente: Elaboración propia a partir de información proporcionada por el MINJUS.

En resumen, se puede concluir que a partir del 2008, las asistencias han ido disminuyendo en términos porcentuales de casi un $67 \%$ a poco más de un $55 \%$, mientras que las inasistencias han subido de $32,6 \%$ a $41,8 \%$.

\begin{tabular}{|c|c|c|c|c|c|c|c|}
\hline \multicolumn{8}{|c|}{$\begin{array}{l}\text { NIVEL DE ASISTENCIA EN CENTROS PUBLICOS Y PRIVADOS CON RELACION AL TOTAL DE CASOS } \\
\text { ATENDIDOS }\end{array}$} \\
\hline AÑOS & Asistencias & $\%$ & Inasistencias & $\%$ & Otros & $\%$ & TOTAL \\
\hline 2008 & 28170 & $66,7 \%$ & 13787 & $32,6 \%$ & 284 & $0,7 \%$ & 42241 \\
\hline 2009 & 40101 & $57,6 \%$ & 28645 & $41,1 \%$ & 894 & $1,3 \%$ & 69640 \\
\hline 2010 & 39847 & $54,7 \%$ & 32004 & $43,9 \%$ & 972 & $1,3 \%$ & 72823 \\
\hline \multirow[t]{2}{*}{2011} & 44735 & $55,1 \%$ & 33926 & $41,8 \%$ & 2498 & $3,1 \%$ & 81159 \\
\hline & 152853 & $57,5 \%$ & 108362 & $40,8 \%$ & 4648 & $1,7 \%$ & 265863 \\
\hline
\end{tabular}

Fuente: Elaboración propia a partir de información proporcionada por el MINJUS.

Esta conclusión puede llevar a cuestionarnos acerca del grado de conocimiento que existiría entre los usuarios del sistema de conciliación, incluyendo a sus abogados, acerca 
de la normatividad vigente y la necesidad de realizar una mayor difusión para que las normas cumplan su finalidad que suponemos ha sido propiciar una mayor concurrencia a las audiencias de conciliación. En resumen, las normas por sí solas no cambian la realidad, es necesario complementarlas con acciones de difusión, entre otras políticas públicas, tarea en la que tanto el Ministerio de Justicia como los mismos centros de conciliación tienen un importante rol que jugar.

\begin{tabular}{|c|c|c|c|c|c|c|c|}
\hline \multicolumn{8}{|c|}{$\begin{array}{l}\text { INASISTENCIAS EN CENTROS DE CONCILIACIÓN PÚBLICOS Y PRIVADOS DIFERENCIADAS POR } \\
\text { SOLICITANTE, INVITADO O AMBOS }\end{array}$} \\
\hline AÑos & Solicitante & $\%$ & Invitado & $\%$ & Ambos & $\%$ & TOTAL \\
\hline 2008 & 2708 & $6,4 \%$ & 6154 & $14,6 \%$ & 4925 & $11,7 \%$ & 13787 \\
\hline 2009 & 2074 & $3,0 \%$ & 21050 & $30,2 \%$ & 5521 & $7,9 \%$ & 28645 \\
\hline 2010 & 2314 & $3,2 \%$ & 23514 & $32,3 \%$ & 6176 & $8,5 \%$ & 32004 \\
\hline \multirow[t]{2}{*}{2011} & 2308 & $2,8 \%$ & 26055 & $32,1 \%$ & 5563 & $6,9 \%$ & 33926 \\
\hline & 9404 & $3,5 \%$ & 76773 & $28,9 \%$ & 22185 & $8,3 \%$ & 108362 \\
\hline
\end{tabular}

Fuente: Elaboración propia a partir de información proporcionada por el MINJUS.

Del total de inasistencias, podemos observar que, como es esperable, la mayor cantidad son generadas por la parte invitada, con un promedio de $28,9 \%$ del total de inasistencias respecto al total de casos atendidos, frente a un 8,3 \% en el que ninguna de las 2 partes asistió y solo 3,5 \% de casos en los que quien faltó fue el solicitante. Un análisis anual, sin embargo, nos muestra un fuerte incremento en el 2009.

INASISTENCIAS EN CENTROS DE CONCILIACIÓN PRIVADOS DIFERENCIADAS POR SOLICITANTE,
\begin{tabular}{|c|r|r|r|r|r|r|c|}
\hline ANTOS & Solicitante & $\%$ & Invitado & $\%$ & Ambos & $\%$ & TOTAL \\
\hline 2008 & 2645 & $8,0 \%$ & 5371 & $16,3 \%$ & 3089 & $9,4 \%$ & 11105 \\
\hline 2009 & 1813 & $3,3 \%$ & 18211 & $33,3 \%$ & 3361 & $6,1 \%$ & 23385 \\
\hline 2010 & 2100 & $3,6 \%$ & 20656 & $35,7 \%$ & 3870 & $6,7 \%$ & 26626 \\
\hline 2011 & 2167 & $3,4 \%$ & 22775 & $35,7 \%$ & 3466 & $5,4 \%$ & 28408 \\
\hline & 8725 & $4,2 \%$ & 67013 & $32,0 \%$ & 13786 & $6,6 \%$ & 89524 \\
\hline
\end{tabular}

Fuente: Elaboración propia a partir de información proporcionada por el MINJUS.

\begin{tabular}{|c|r|r|r|r|r|r|r|}
\hline \multicolumn{6}{|c|}{ INASISTENCIAS EN CENTROS PÚBLICOS POR PARTE (SOLICITANTE/ASISTENTE/AMBOS) } \\
\hline ANNOS & Solicitante & \multicolumn{1}{c}{$\%$} & Invitado & \multicolumn{1}{c}{$\%$} & \multicolumn{1}{c|}{ Ambos } & \multicolumn{1}{c|}{ TOTAL } \\
\hline 2008 & 63 & $0,7 \%$ & 783 & $8,4 \%$ & 1836 & $19,8 \%$ & 2682 \\
\hline 2009 & 261 & $1,7 \%$ & 2839 & $19,0 \%$ & 2160 & $14,4 \%$ & 5260 \\
\hline 2010 & 214 & $1,4 \%$ & 2858 & $19,0 \%$ & 2306 & $15,3 \%$ & 5378 \\
\hline 2011 & 141 & $0.8 \%$ & 3280 & $19,0 \%$ & 2097 & $12,1 \%$ & 5518 \\
\hline
\end{tabular}

Fuente: Elaboración propia a partir de información proporcionada por el MINJUS. 
Comparando los datos de los centros privados y los centros públicos, se observa que en los centros privados la inasistencia del invitado es más marcada que en centros públicos $(38,6 \%$ versus $32,1 \%),{ }^{7}$ mientras que en el caso del solicitante, la situación es inversa, con mayores inasistencias en los centros públicos que en los centros privados (16\% versus 10,8\%). ${ }^{8}$

La mayor ausencia del invitado en comparación con el solicitante se explicaría por el hecho de que el solicitante es, en teoría, el interesado en llegar a una conciliación, además de ser el que suele cubrir el costo del servicio (en el caso de los centros privados). En los casos de inasistencia del solicitante, quizás se deba a que considera a la conciliación como un mero trámite, lo que explicaría por qué en los centros privados el porcentaje de inasistencias del solicitante es menor (ahorro en el costo de este proceso).

\section{ANÁLISIS DE LA INFORMACIÓN ESTADÍSTICA EN MATERIA CIVIL}

\begin{tabular}{|c|c|c|c|}
\hline \multicolumn{2}{|c|}{ ASISTENCIAS E INASISTENCIAS EN MATERIA CIVIL EN CENTROS PRIVADOS } \\
\hline ANNOS & $\begin{array}{c}\text { Casos con asistencia de ambas } \\
\text { partes }\end{array}$ & $\begin{array}{c}\text { Casos con inasistencia de una o } \\
\text { ambas partes }\end{array}$ & Otros* $^{*}$ \\
\hline 2001 & $35,5 \%$ & $62,6 \%$ & $2,0 \%$ \\
\hline 2002 & $30,7 \%$ & $68,1 \%$ & $1,2 \%$ \\
\hline 2003 & $32,3 \%$ & $66,9 \%$ & $0,8 \%$ \\
\hline 2004 & $31,6 \%$ & $67,7 \%$ & $0,7 \%$ \\
\hline 2005 & $28,3 \%$ & $71,1 \%$ & $0,6 \%$ \\
\hline 2006 & $34,9 \%$ & $64,6 \%$ & $0,5 \%$ \\
\hline 2007 & $45,7 \%$ & $53,7 \%$ & $0,7 \%$ \\
\hline 2008 & $62,3 \%$ & $36,9 \%$ & $0,7 \%$ \\
\hline 2009 & $50,0 \%$ & $48,8 \%$ & $1,0 \%$ \\
\hline 2010 & $45,3 \%$ & $53,3 \%$ & $1,3 \%$ \\
\hline 2011 & $46,8 \%$ & $52,3 \%$ & $1,0 \%$ \\
\hline
\end{tabular}

Fuente: Elaboración propia a partir de datos MINJUS

* El rubro "Otros" se refiere a los casos que se archivaron por desconocimiento del domicilio, por decisión fundamentada del conciliador o por informes.

Si bien vemos que en el año 2008 el porcentaje de asistencia de ambas partes aumenta a $62,3 \%$ (de $45,7 \%$ en el 2007), se observa también que esta cifra decrece nuevamente en el año 2009 y aún más en el 2010 y el 2011, hasta casi retroceder al nivel del 2007.

Sumando inasistencias del solicitante únicamente e inasistencias de ambas partes.

8 Sumando inasistencias del invitado únicamente e inasistencias de ambas partes. 
En líneas generales, en su gran mayoría es solo una de las partes la que no asiste al proceso. Solo en un porcentaje minoritario de casos, ninguna de las dos partes acude a las audiencias convocadas, como se aprecia en el siguiente cuadro, porcentaje que además va disminuyendo en los últimos años, hasta llegar a menos de $6 \%$ en el 2011:

\begin{tabular}{|c|c|c|c|}
\hline \multicolumn{2}{|c|}{$\begin{array}{c}\text { PORCENTAJE DE CASOS RESUELTOS POR INASISTENCIA EN MATERIA CIVIL SEGÚN TIPO DE } \\
\text { INASISTENCIA }\end{array}$} \\
\hline ANNOS & Inasistencia de una de las partes & Inasistencia de ambas partes & TOTAL \\
\hline 2001 & $49,8 \%$ & $12,7 \%$ & $62,5 \%$ \\
\hline 2002 & $57,7 \%$ & $10,3 \%$ & $68,0 \%$ \\
\hline 2003 & $55,4 \%$ & $11,5 \%$ & $66,9 \%$ \\
\hline 2004 & $57,0 \%$ & $10,7 \%$ & $67,7 \%$ \\
\hline 2005 & $61,0 \%$ & $10,1 \%$ & $71,1 \%$ \\
\hline 2006 & $54,4 \%$ & $10,2 \%$ & $64,6 \%$ \\
\hline 2007 & $45,3 \%$ & $8,4 \%$ & $53,8 \%$ \\
\hline 2008 & $26,8 \%$ & $10,2 \%$ & $36,9 \%$ \\
\hline 2009 & $42,1 \%$ & $6,7 \%$ & $48,8 \%$ \\
\hline 2010 & $45,9 \%$ & $7,4 \%$ & $53,3 \%$ \\
\hline 2011 & $46,4 \%$ & $5,9 \%$ & $52,3 \%$ \\
\hline
\end{tabular}

Fuente: Elaboración propia a partir de la información brindada por el MINJUS.

En tercer lugar, analizaremos cómo se resolvieron los casos en los que sí se contó con asistencia de ambas partes en relación con el total de casos. Si bien el porcentaje de casos concluidos por acuerdo total no es, en general, muy alto con respecto al total de ingresos, se nota una tendencia al aumento de estos casos a partir del año 2006, pasando de un $21 \%$ en promedio durante el primer lustro de instalado el sistema (2001-2005) a un 26,9 \%, en el año 2006. El año 2007, el aumento de casos concluidos por acuerdo total es más notorio aún, llegando a un 39,7 \%, llegando a su tope en el 2008, con más de la mitad del total de casos atendidos que alcanzaron un acuerdo total. A partir de ese año, el porcentaje de casos con acuerdo total vuelve a caer, bajando hasta menos de 1 de cada 3 en el 2011. Lamentablemente, el porcentaje de casos con falta de acuerdo también aumentó a partir del 2007 (fecha en la que había descendido hasta apenas 5,6 \%) a 14,3\% en el 2011, el porcentaje más alto desde el inicio de la institución conciliatoria. 


\begin{tabular}{|c|c|c|c|c|}
\hline \multicolumn{5}{|c|}{$\begin{array}{c}\text { MODALIDAD DE CONCLUSIÓN EN LOS CASOS CON ASISTENCIA EN RELACIÓN AL TOTAL DE } \\
\text { CASOS ATENDIDOS EN MATERIA CIVIL }\end{array}$} \\
\hline ANNOS & ACUERDO TOTAL & ACUERDO PARCIAL & FALTA DE ACUERDO & TOTAL \\
\hline 2001 & $21,4 \%$ & $1,4 \%$ & $12,7 \%$ & $35,5 \%$ \\
\hline 2002 & $20,5 \%$ & $0,8 \%$ & $9,4 \%$ & $30,7 \%$ \\
\hline 2003 & $22,4 \%$ & $0,6 \%$ & $9,3 \%$ & $32,3 \%$ \\
\hline 2004 & $21,5 \%$ & $1,8 \%$ & $8,3 \%$ & $31,6 \%$ \\
\hline 2005 & $20,6 \%$ & $0,7 \%$ & $7,0 \%$ & $28,3 \%$ \\
\hline 2006 & $26,9 \%$ & $0,4 \%$ & $7,6 \%$ & $34,9 \%$ \\
\hline 2007 & $39,7 \%$ & $0,4 \%$ & $5,6 \%$ & $45,7 \%$ \\
\hline 2008 & $51,7 \%$ & $0,6 \%$ & $10,0 \%$ & $62,4 \%$ \\
\hline 2009 & $36,8 \%$ & $0,5 \%$ & $12,8 \%$ & $49,8 \%$ \\
\hline 2010 & $32,3 \%$ & $0,4 \%$ & $12,6 \%$ & $45,3 \%$ \\
\hline 2011 & $32,1 \%$ & $0,4 \%$ & $14,3 \%$ & $46,3 \%$ \\
\hline
\end{tabular}

Fuente: Elaboración propia a partir de la información brindada por el MINJUS.

\section{ANÁLISIS DE INFORMACIÓN ESTADÍSTICA EN MATERIA DE FAMILIA}

\begin{tabular}{|c|c|c|c|}
\hline \multicolumn{5}{|c|}{$\begin{array}{c}\text { PORCENTAJE DE ASISTENCIA A LAS AUDIENCIAS EN MATERIA FAMILIA EN CENTROS DE } \\
\text { CONCILIACIÓN PRIVADOS }\end{array}$} \\
\hline \multirow{2}{*}{ ANNOS } & $\begin{array}{c}\text { Casos con asistencia de ambas } \\
\text { partes }\end{array}$ & $\begin{array}{c}\text { Casos con inasistencia de una o } \\
\text { ambas partes }\end{array}$ & Otros \\
\hline 2001 & $64,4 \%$ & $33,0 \%$ & $2,6 \%$ \\
\hline 2002 & $67,8 \%$ & $30,9 \%$ & $1,3 \%$ \\
\hline 2003 & $69,1 \%$ & $30,1 \%$ & $0,8 \%$ \\
\hline 2004 & $72,8 \%$ & $26,8 \%$ & $0,4 \%$ \\
\hline 2005 & $78,1 \%$ & $21,5 \%$ & $0,4 \%$ \\
\hline 2006 & $79,3 \%$ & $20,5 \%$ & $0,3 \%$ \\
\hline 2007 & $78,9 \%$ & $20,7 \%$ & $0,4 \%$ \\
\hline 2008 & $82,3 \%$ & $17,0 \%$ & $0,6 \%$ \\
\hline 2009 & $77,0 \%$ & $21,8 \%$ & $1,2 \%$ \\
\hline 2010 & $77,8 \%$ & $21,4 \%$ & $0,7 \%$ \\
\hline 2011 & $76,5 \%$ & $21,9 \%$ & $1,6 \%$ \\
\hline
\end{tabular}

Fuente: Elaboración propia a partir de datos MINJUS.

* El rubro "Otros" se refiere a los casos que se archivaron por desconocimiento del domicilio, por decisión fundamentada del conciliador e informes del conciliador. 
A diferencia de los casos en materia civil, en materia de familia el número de asistencias es mucho más alto. El año en que se registró el más bajo porcentaje de asistencia fue el 2001, con un $64,4 \%$, porcentaje que luego fue incrementando año a año, llegando a su pico en el 2008, año en que se refleja un ligero descenso, llegando a un 76,5\% en el 2011.

\begin{tabular}{|c|c|c|c|}
\hline \multicolumn{4}{|c|}{$\begin{array}{c}\text { PORCENTAJE DE ASISTENCIA A LAS AUDIENCIAS EN MATERIA DE FAMILIA EN CENTROS DE } \\
\text { CONCILIACIÓN PÚBLICOS }\end{array}$} \\
\hline \multirow{2}{*}{ AÑOS } & $\begin{array}{c}\text { Casos con asistencia de ambas } \\
\text { partes }\end{array}$ & $\begin{array}{c}\text { Casos con inasistencia de una } \\
\text { o ambas partes }\end{array}$ & Otros \\
\hline 2008 & $71,9 \%$ & $27,7 \%$ & $0,4 \%$ \\
\hline 2009 & $66,0 \%$ & $32,3 \%$ & $1,7 \%$ \\
\hline 2010 & $65,2 \%$ & $33,3 \%$ & $1,5 \%$ \\
\hline 2011 & $61,2 \%$ & $28,9 \%$ & $10,0 \%$ \\
\hline
\end{tabular}

Fuente: Elaboración propia a partir de datos MINJUS.

En los centros de conciliación públicos, el porcentaje de casos con asistencia de ambas partes es ligeramente inferior en comparación al de los centros de conciliación privados. Al igual que en estos, durante el período analizado se observa un descenso progresivo del porcentaje de casos con asistencia de ambas partes, aunque esto no se traduce en un porcentaje mayor de casos con inasistencia de alguna de las partes, sino que en el año 2011 destaca un atípico alto porcentaje de casos clasificados como otros, que se refieren a casos que concluyeron por desconocimiento del domicilio o por decisión fundamentada o informe del conciliador.

\begin{tabular}{|c|c|c|c|}
\hline \multicolumn{3}{|c|}{$\begin{array}{c}\text { PORCENTAJE DE ASISTENCIA A LAS AUDIENCIAS EN MATERIA FAMILIA EN CENTROS DE } \\
\text { CONCILIACIÓN PÚBLICOS MÁS PRIVADOS }\end{array}$} \\
\hline AÑOS & $\begin{array}{c}\text { Casos con asistencia de ambas } \\
\text { partes }\end{array}$ & $\begin{array}{c}\text { Casos con inasistencia de una } \\
\text { o ambas partes }\end{array}$ & Otros \\
\hline 2008 & $76,1 \%$ & $23,3 \%$ & $0,5 \%$ \\
\hline 2009 & $71,4 \%$ & $27,1 \%$ & $1,4 \%$ \\
\hline 2010 & $71,5 \%$ & $27,4 \%$ & $1,1 \%$ \\
\hline 2011 & $69,1 \%$ & $25,2 \%$ & $5,7 \%$ \\
\hline Promedio & $71,3 \%$ & $26,1 \%$ & $2,6 \%$ \\
\hline
\end{tabular}

Fuente: Elaboración propia a partir de datos MINJUS.

En resumen, en los últimos 4 años, 7 de cada 10 casos recibidos por los centros de conciliación públicos y privados en materia de familia concluyeron con asistencia de ambas partes; 1 de 4 concluyeron con inasistencia de al menos una de las partes, y un porcentaje minoritario concluyó por otros motivos (desconocimiento del domicilio o por decisión fundamentada e informe del conciliador). 


\begin{tabular}{|c|c|c|c|}
\hline \multicolumn{4}{|c|}{$\begin{array}{l}\text { PORCENTAJE DE CASOS POR MODALIDAD DE RESOLUCIÓN EN MATERIA DE FAMILIA EN } \\
\text { CENTROS PRIVADOS }\end{array}$} \\
\hline AÑOS & Acuerdo Total & Acuerdo Parcial & Falta de acuerdo \\
\hline 2001 & $48,3 \%$ & $6,9 \%$ & $9,2 \%$ \\
\hline 2002 & $54,2 \%$ & $2,3 \%$ & $11,3 \%$ \\
\hline 2003 & $59,0 \%$ & $1,7 \%$ & $8,4 \%$ \\
\hline 2004 & $63,1 \%$ & $1,1 \%$ & $8,6 \%$ \\
\hline 2005 & $69,0 \%$ & $0,6 \%$ & $8,5 \%$ \\
\hline 2006 & $72,0 \%$ & $1,1 \%$ & $6,2 \%$ \\
\hline 2007 & $72,1 \%$ & $0,8 \%$ & $6,0 \%$ \\
\hline 2008 & $72,5 \%$ & $1,2 \%$ & $8,6 \%$ \\
\hline 2009 & $62,6 \%$ & $1,8 \%$ & $12,6 \%$ \\
\hline 2010 & $65,3 \%$ & $1,2 \%$ & $11,3 \%$ \\
\hline 2011 & $64,0 \%$ & $1,0 \%$ & $11,5 \%$ \\
\hline
\end{tabular}

Fuente: Elaboración propia a partir de datos MINJUS.

En materia de familia en centros privados, es interesante observar los elevados porcentajes de casos en los que se arriba a acuerdo total, frente a la ínfima cantidad en la que se llega a acuerdo parcial o no se llega a acuerdo. Se nota una tendencia ascendente entre el 2001 al 2006, que es seguida de un período de estabilidad hasta el 2008, a partir de cuando nuevamente se registra un ligero descenso en el porcentaje de casos con acuerdo total.

Los casos finalizados por falta de acuerdo en materias familiares en centros privados en ningún año han superado el 12,6 \% del total de casos, que fue el máximo porcentaje que se dio en el año 2009. En general, se puede apreciar que los porcentajes de casos que han concluido por falta de acuerdo en materia familiar en centros privados son bastante bajos, dato que resulta bastante positivo.

\begin{tabular}{|c|c|c|c|}
\hline \multicolumn{4}{|c|}{$\begin{array}{c}\text { PORCENTAJE DE CASOS POR MODALIDAD DE RESOLUCIÓN EN MATERIA DE FAMILIA EN } \\
\text { CENTROS PÚBLICOS }\end{array}$} \\
\hline AÑOS & Acuerdo Total & Acuerdo Parcial & Falta de acuerdo \\
\hline 2008 & $59,4 \%$ & $1,0 \%$ & $11,5 \%$ \\
\hline 2009 & $51,4 \%$ & $1,5 \%$ & $13,1 \%$ \\
\hline 2010 & $50,1 \%$ & $1,6 \%$ & $13,5 \%$ \\
\hline 2011 & $45,3 \%$ & $1,6 \%$ & $14,3 \%$ \\
\hline Promedio & $50,4 \%$ & $1,4 \%$ & $13,3 \%$ \\
\hline
\end{tabular}

Fuente: Elaboración propia a partir de datos MINJUS. 
En la misma línea, los centros públicos también han mostrado un buen desempeño, teniendo un promedio de 50,4\% de casos con acuerdo total entre los años 2008 y 2011; $1,4 \%$ de casos con acuerdo parcial, y 13,3\% concluidos con falta de acuerdo en los mismos años. Esto con relación al total de casos ingresados. Recordemos que un 71,9 \% de los casos asistieron, por lo que es solo sobre esa base que hubo posibilidad de llegar a acuerdo.

\begin{tabular}{|c|c|c|c|}
\hline AÑ̃os & Acuerdo Total & Acuerdo Parcial & Falta de acuerdo \\
\hline 2008 & $64,7 \%$ & $1,1 \%$ & $10,3 \%$ \\
\hline 2009 & $56,9 \%$ & $1,6 \%$ & $12,8 \%$ \\
\hline 2010 & $57,7 \%$ & $1,4 \%$ & $12,4 \%$ \\
\hline 2011 & $55,0 \%$ & $1,3 \%$ & $12,8 \%$ \\
\hline Promedio & $57,6 \%$ & $1,4 \%$ & $12,4 \%$ \\
\hline
\end{tabular}

Fuente: Elaboración propia a partir de datos MINJUS.

En resumen, del total de casos en los que los centros de conciliación sí tuvieron alguna oportunidad de promover acuerdos en materia de familia, consiguieron su objetivo en 4 de cada 5 casos, mientras que en menos de 1 de cada 5 casos no pudieron arribar a acuerdos ni siquiera parciales. Estos resultados son bastante auspiciosos. 


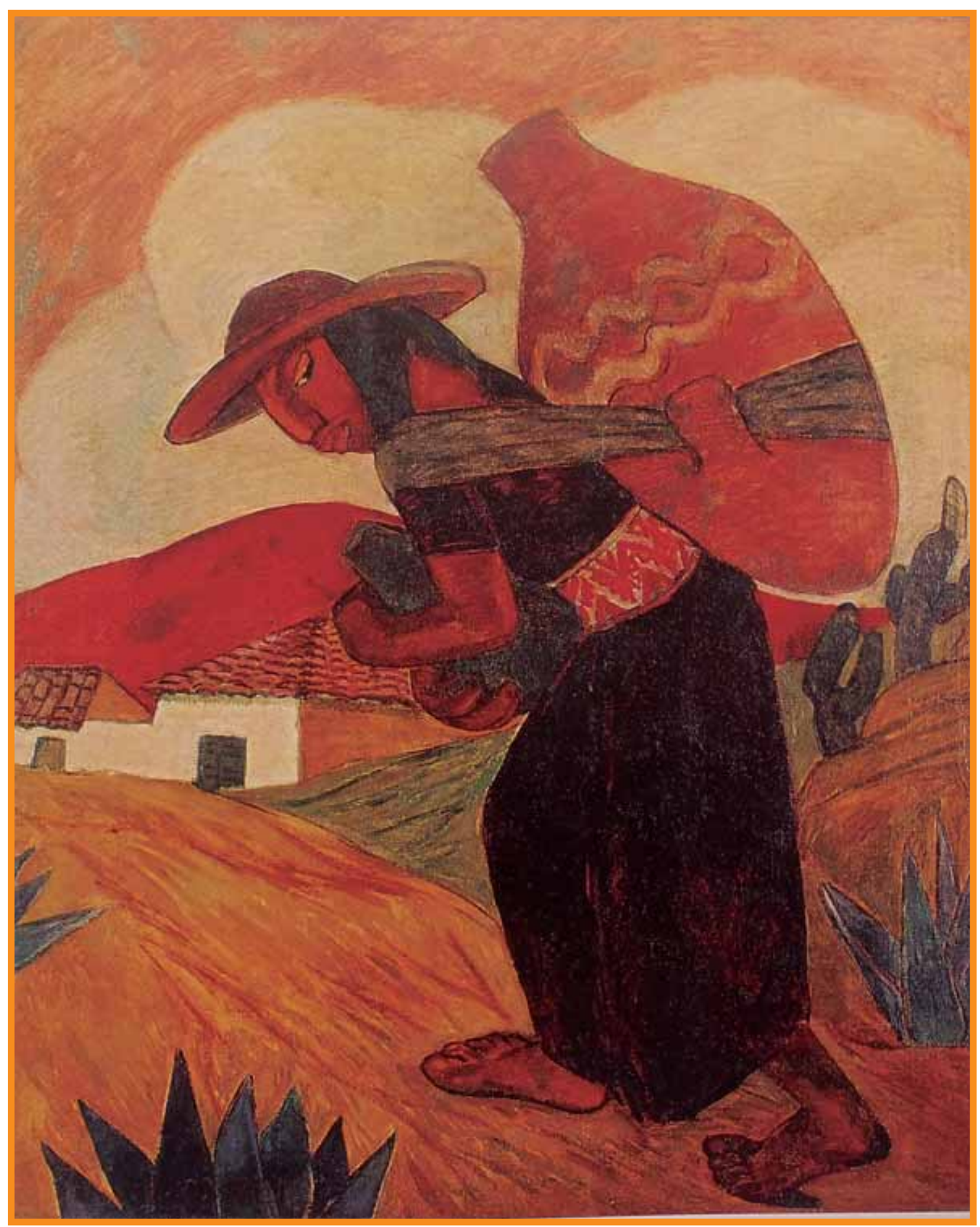

India huanca. 